\title{
La enseñanza tradicional de las ciencias versus las nuevas tendencias educativas
}

\author{
María Isabel Torres Salas ${ }^{1}$ \\ División de Educología del Centro de Investigación y Docencia en \\ Educación (CIDE), Universidad Nacional \\ Heredia, Costa Rica
}

Recibido 27 de octubre de 2009 • Aceptado 02 de diciembre de 2009

\begin{abstract}
Resumen. Para pensar en una propuesta educativa que enseñe a aprender, es necesario pensar en un cambio no sólo en lo educativo, sino, también, en lo político, económico, social, ecológico y cultural, entre otros; que permita una comprensión de la realidad y es aquí donde la construcción del conocimiento y el rol de las ciencias tienen un papel fundamental. Pero no debemos olvidar que el desarrollo de la ciencia ha estado marcado por la llamada ciencia positivista, la cual se caracteriza por interpretar los fenómenos y la forma cómo funcionan éstos por medio de teorías y leyes, en los que el contexto y el ser humano tienen un papel protagónico muy pobre, por no decir ninguno; a esto se le puede llamar el cientificismo, que ha permitido un desarrollo, incluso, sobre de las necesidades humanas. Pero, desde los 90s, se da un resurgimiento paulatino del humanismo en los campos educativos y se busca trabajar en la revalorización de lo humano, lo cual implica una serie de cambios en el plano epistemológico y metodológico que nos impulsa hacia nuevas formas de trabajar. Esto nos llama a reflexionar en alternativas trascendentales para la construcción del conocimiento, que superen la enseñanza tradicional de las ciencias, que sean amplias, sistemáticas, flexibles y enmarcadas en una cultura humanista. Algunos modelos de las nuevas tendencias son: investigación dirigida, aprendizaje por descubrimiento, aprendizaje por indagación y la enseñanza de las ciencias y las nuevas tecnologías.
\end{abstract}

Palabras clave. Alfabetización científica, didáctica, enseñanza, aprendizaje, ciencias, tecnologías.

\footnotetext{
${ }^{1}$ Máster en Psicopedagogía, se he desempeñado como profesora de Química en Enseñanza Media, Asesora en el Centro Nacional de Didáctica, del Ministerio de Educación Pública (MEP), Costa Rica, Asesora en el Ministerio de Ciencia y Tecnología, Costa Rica, Vicedecana del Centro de Investigación y Educación de la Universidad Nacional (UNA), Costa Rica. Trabaja como académica en Educología del Centro de Investigación y Docencia en Educación (CIDE) de la Universidad Nacional (UNA), Costa Rica. Principales publicaciones: La enseñanza y el aprendizaje de la química con una metodología activa, Actividades complementarias a los programas de estudio de ciencias para séptimo, octavo y noveno año, Actividades complementarias de estudio de décimo año, Propuesta Interdisciplinaria de Carta de la Tierra para cuatro disciplinas de sétimo año de la Educación General Básica. Correo electrónico: isabeltorrescr@yahoo.com
}

La Revista Electrónic@ Educare está indizada en el Catálogo LATINDEX.

Artículo protegido por la licencia Creative Commons. 
Abstract. To think of an educational proposal that teaches how to learn, it is necessary to consider a change not only educationally but also political, social, economical, ecological, cultural, among others, to enable an understanding of reality and in which there can be a construction of knowledge and a crucial role of sciences. But we must not forget that the development of science has been marked by the so-called positivistic science that it is characterized by interpreting phenomena and how this function through theories and laws, where the context and humans have a very poor leading role, if any, to which one can call scientism, which has allowed development even above human needs. However, since the $90 \mathrm{~s}$, there is a resurgence of progressive humanism in the educational fields, where there is a search of a revaluation of what it is considered human, which involves a series of epistemological and methodological changes that drives us towards new ways of working. This calls us to reflect on extreme choices to build knowledge, beyond the traditional teaching of the sciences, which are comprehensive, systematic, and flexible and rooted in a humanistic culture. Some models of the new trends are: directed research, discovery learning, inquiry learning and teaching of science and new technologies.

Key words. Scientific literacy, didactic, education and learning, sciences, technologies.

\section{Introducción}

Los retos que recibe la educación han sido enfrentados, durante las últimas décadas del siglo xx e inicios del xxI, con la búsqueda de un modelo holístico, endógeno y sostenible. Sin embargo, las tentativas de llevar a la realidad estas iniciativas, con sensibilidad ambiental y rostro humano, no han dado los resultados esperados. Los actuales contextos de un mundo globalizado expresan las grandes diferencias, que evidencian el fracaso de las políticas de erradicación del hambre y de construcción de una paz duradera. Ante esto, la educación, una de las responsables de comunicar los conocimientos, permanece ciega ante lo que es el conocimiento humano, sus disposiciones, sus imperfecciones, sus dificultades, sus tendencias tanto al error como a la ilusión, y no se preocupa, en absoluto, por hacer conocer lo que es conocer (Morin, 2000).

Para pensar en una propuesta educativa que enseñe a "aprender a aprender", es necesario pensar en un cambio no sólo en lo educativo, sino también en lo político, económico, social, ecológico, espiritual y cultural, entre otros; que permita una comprensión de la realidad. En esto, la construcción del conocimiento y el rol de las ciencias tienen un papel fundamental, para lo cual debemos pensar en formar un ser humano como objeto de conocimiento individual y social. En este sentido, pensadores como Freire (2002) proponen que es necesario construir una epistemología sobre la base de una comprensión integral del ser, partiendo desde el propio conocimiento, ya que la realidad de los otros se entiende sólo cuando se percibe y entiende la propia realidad.

Sin embargo, para comprender el rol protagónico que deben tener las ciencias en la construcción de una sociedad sostenible, dispuesta a respetar las diversas formas de vida, a cuidar la naturaleza, a promover la justicia económica y a fundar una cultura de paz, es necesario entender el desarrollo histórico que éstas han tenido en el aporte del conocimiento y, más aún, el por qué la didáctica de las ciencias ha sido dominada, casi en su totalidad, por el positivismo.

\section{Historia del desarrollo de las ciencias y la enseñanza tradicional}

Si lleváramos a cabo un recorrido rápido por la historia, podríamos ver que durante la Edad Media, la enseñanza de las ciencias fue mínima, tanto en escuelas como en colegios y universidades. Durante el Renacimiento, las corrientes humanistas llegaron a los sistemas educativos, pero no así las 
ciencias, pues no fue hasta el siglo XVIII y parte del XIX, con el auge de los grandes descubrimientos, que se comenzó a despertar el interés por ellas. Sin embargo, en las instituciones educativas, la enseñanza de las ciencias tenía poca importancia y, además, se hacía de forma teórica, ya que la enseñanza experimental de estas disciplinas llegó aún más tarde.

Todo este desarrollo de la ciencia estuvo marcado por la llamada ciencia positivista, la cual se caracteriza por interpretar los fenómenos y la forma cómo funcionan por medio de teorías y leyes, en los que el contexto y el ser humano tienen un papel protagónico muy pobre, por no decir ninguno; a esto se le puede llamar el cientificismo, es decir, el desarrollo científico-técnico se valora por encima, incluso, de las necesidades humanas, las cuales, se supone, tratan de satisfacer sin justificación alguna (Diéguez, 1993). Esto ha permitido dominar los acontecimientos tanto del mundo físico como del social, partiendo de la premisa de que todo lo que se puede constatar es positivo y relativo; en cambio lo no demostrable, es absoluto y ambiguo.

Para Feyerabend (citado por Toledo, 1998), los científicos han esculpido la realidad del mundo utilizando el saber-hacer científico del que somos parte, ignorando el lado subjetivo del conocimiento, éste último entrelazado de maneras complejas con diversas manifestaciones materiales. Esta posición epistemológica implica, de forma tácita, la primacía del objeto y la omisión o desprecio de la actividad del sujeto como determinante del conocimiento (Núñez, 2000). Desde este planteamiento, el trabajo de la ciencia consiste o se reduce a la aplicación del método científico, es decir, recoger datos, observar, analizar, experimentar para llegar a conclusiones mediante la utilización de procedimientos lógicos, extraídos de las mismas teorías y leyes, que los respaldan.

Según Tejada (2005), esta visión de la ciencia está muy ligada a la teoría empirista e inductivista de Bacon, Hume, Comte o Mach, como posteriormente se ha demostrado; incluso el mismo Popper es crítico de ello, y se establece en dos supuestos falsos: en primer lugar, la inducción no se puede justificar sobre bases estrictamente lógicas, es decir, de muchos enunciados singulares no puede derivarse una teoría, podría darse el caso de que las premisas sean verdaderas y que la conclusión sea falsa y, en segundo lugar, la creencia de que la ciencia se inicia con la observación y que ésta es una base objetiva y segura de ella, sin considerar que el observador ve e interpreta de acuerdo con sus conocimientos, experiencias, expectativas y, en última instancia, con su historia de vida. En ese acontecer, Popper introduce una nueva variante sobre el progreso del conocimiento, la cual ha sido relevante por sus consecuencias: el falsacionismo frente a la verificación, ya que se ha demostrado que las teorías científicas no pueden ser validadas por las observaciones particulares, pero sí pueden ser negadas o, al menos, refutadas por éstas.

De acuerdo con lo anterior, para los positivistas, el conocimiento científico es un conjunto de derivaciones lógicas y contrastaciones empíricas, en las que lo primordial es la coherencia interna y la correspondencia de las construcciones formales autónomas con la marcha y las características de los hechos reales. Pero, como la realidad no es independiente de la historia ni el ámbito social del sujeto, el conocimiento científico posee una insoslayable dimensión institucional y como institución social la ciencia se encuentra sometida a la complejidad de relaciones condicionantes entre las diversas instituciones del sistema social (Pérez Gómez, 1978, citado por Tejada, 2005), es decir, el sujeto y la sociedad investigan influidos por paradigmas y plataformas cognoscitivas históricamente gestadas y socialmente condicionadas dentro de un sistema de relaciones. Pero, según Huffmann (2005), el conflicto epistemológico es delimitar y precisar los hechos reales que determinan el desarrollo del conocimiento, que lo benefician o lo dificultan.

En el proceso histórico de las décadas de los 60s y 70s surgieron, en el campo de la filosofía de las ciencias, varias concepciones epistemológicas con respecto a la construcción y evolución del conocimiento científico, por encima de las posturas positivistas que se habían venido dando. 
Los aportes de los programas de investigación de Lakatos y los trabajos de Kuhn y Feyerabend contribuyeron a la construcción de nuevos modos de hacer y pensar la ciencia en consonancia con una postura del ser humano como sujeto-objeto de conocimiento científico. Se puede afirmar que desde los 90s, se da un resurgimiento paulatino del humanismo en los campos educativos, y se busca trabajar en la revalorización de lo humano, lo cual implica una serie de cambios en los planos epistemológico y metodológico y, finalmente, nos conduce a una revisión de nuestros cimientos convencionales de hacer ciencia y nos impulsa hacia nuevas formas de trabajar, más consecuentes con la posición de la unidad en diversidad sujeto-objeto del conocimiento y sus inseparables e interdependientes vínculos con el entorno natural, cultural y social que lo rodea.

Esto nos llama a reflexionar en opciones trascendentales para la construcción del conocimiento, que superen la enseñanza tradicional de las ciencias, que sean amplias, sistemáticas, flexibles y enmarcadas dentro de una cultura humanista. Según Morin (2001), esta cultura de construcción del conocimiento debe ser genérica, alimentar la inteligencia en general, enfrentar las grandes interrogantes humanas, estimular la reflexión sobre el saber y favorecer la integración personal de los conocimientos para formar personas críticas, responsables de su aprendizaje y de su actuación. En la actualidad, se habla del enfoque ecológico, también conocido como "Emergente" en el campo pedagógico -que supera al positivismo, enfrentado por la línea hermenéutica dialéctica del siglo XIX- y es compatible con la teoría crítica, la cual, desde 1930, empezó a señalar las limitaciones del positivismo. El paradigma ecológico que nace como un modelo sociológico viene a proporcionar a la enseñanza y al aprendizaje de las ciencias en particular una propuesta de trasformación y praxis escolar, que permite trabajar con ciertos principios epistemológicos para proporcionar las bases teóricas-metodológicas, en las que se sustenta la concepción de conocimiento: La realidad es un proceso de construcción socio-histórico cultural, en la que intervienen la historicidad del sujeto y la intencionalidad del conocimiento, la creatividad, la dimensión antropológica y la dimensión axiológica.

\section{Nuevas tendencias en la enseñanza de las ciencias}

Las nuevas generaciones nacen, crecen y son educadas en un contexto tecnológico, invadido por lo global, pero para que esto no se convierta en un aspecto negativo, el punto de partida de la educación debe contemplar el desarrollo sociohistórico local del individuo, sin que por esto se rechace lo global. Decía Freire que lo local y lo global son como dos pies con los cuales nuestro pensamiento debería caminar en un mundo cambiante, en el que sabemos que la revolución tecnológica ha contribuido mucho a estos cambios acelerados. Por tanto, debemos ser conscientes de que las formas de interacción y comunicación entre las personas y los escenarios, condicionan los aprendizajes inmediatos y futuros.

En los últimos años, las estrategias de aprendizaje han ido cobrando una importancia cada vez mayor, tanto en la investigación psicológica como en la práctica educativa, que ha venido a convertir el aprender a aprender en una de las metas fundamentales de cualquier proyecto educativo (Pozo y Monereo, 1999). Por ello, los docentes debemos tener claridad que la enseñanza de las ciencias no escapa a esta realidad y, sobre todo, tener conciencia de que las directrices que orientan nuestra práctica profesional, deben acoplarse a las necesidades de la diversidad y de la complejidad. Por tanto, el docente debe ver el hecho educativo como un propósito de construcción de sujetos, diversos y creativos en sociedades complejas. Desde esta concepción, el docente consolida las actuaciones por medio de su reflexionar y comprender la realidad educativa, con lo que promueve el mantenimiento de su actividad profesional, en la formación humana. Morin dice que hay que 
crear y formar seres humanos "con ciencia, pero también con conciencia”, y podría decirse que esta afirmación constituye la principal tarea de la enseñanza de las ciencias contemporáneas.

Enseñar ciencias de forma contextualizada y relacionada con la vida cotidiana es uno de los retos más desafiantes de esta época. Son muchos los métodos y las técnicas que los docentes aplican para enseñar esta disciplina, pero algunas de ellas están muy apegadas a la herencia que nos ha dejado el positivismo, y se utiliza el método científico como el único instrumento para llegar al conocimiento. En otros casos, la principal forma de enseñarla es en el papel, como un conjunto de hechos y verdades estables e incuestionables, que el libro de texto contiene, que el profesor, supuestamente, sabe y que el alumno tiene que memorizar, para poder contestar las preguntas a las que es sometido en los exámenes. Esto ocurre, según Núñez (2000), a pesar de la influencia de diferentes corrientes que proponen la utilización de múltiples formas de enseñar las ciencias, en las que se incentiva la realización de experimentos y demostraciones en la clase, observaciones en el campo con la participación activa de los estudiantes en los procesos de enseñanza y de aprendizaje, con el fin de construir los conocimientos en forma conjunta, contextualizados y ligados a la actividad diaria de las personas.

Dentro de estas tendencias, se le da mucha importancia al fin con que puede utilizarse el conocimiento científico, ya que puede ser benéfico o perjudicial para la humanidad, para el medio ambiente y para el planeta en su totalidad (Núñez, 2000). Se hace hincapié en que los recursos naturales no son infinitos; además, se considera de gran importancia enseñar a los alumnos a tomar decisiones razonadas, y que comprendan que la enseñanza de la ciencia tiene como propósito la preparación de los ciudadanos, para que participen reflexivamente, de manera informada y responsable en la solución de problemas sociales y personales (Huffmann, 2005).

Algunos modelos empleados para la enseñanza de las ciencias, según las nuevas tendencias, se analizan a continuación.

\section{Investigación dirigida}

El concepto de aprendizaje como un proceso de investigación no es nuevo; sin embargo, durante la década de los 90s y principios de este siglo, han surgido iniciativas afines con ese planteamiento y ha adquirido un impulso importante, especialmente fundamentado en el constructivismo. Podría decirse que la investigación dirigida es una metodología sustentada en el paradigma naturalista, fortalecido con la teoría del constructivismo, que propone el aprendizaje de los conceptos como una construcción de manera activa por parte del aprendiz y la cual se lleva a cabo con base en en los conocimientos previos (Pozo y Gómez, 1998).

La investigación dirigida plantea el aprendizaje de la ciencia como un proceso de construcción social de teorías y de modelos, los docentes se convierten en guías para que sus alumnos logren cambios tanto en los conceptos, como en las actitudes y en los procedimientos, lo que permite un mayor desarrollo cognitivo que los faculte para resolver problemas teóricos y prácticos. De acuerdo con Diego-Rasilla (2004), la utilización de la investigación dentro de un aula implica, necesariamente, la puesta en práctica del pensamiento científico, por lo que es un modo de indagar en la realidad.

Esta forma de aprendizaje acerca al estudiante al proceso sociohistórico y a la situación problematizadora en la que se generó el conocimiento. Este proceso no se da mediante las estrategias tradicionales de enseñanza de las ciencias, ya que el énfasis está dirigido a la justificación de los fenómenos y deja de lado la contextualización o vinculación con la realidad cotidiana del fenómeno (Campanario y Moya, 2002). 
Furió y Guisasola (1999) plantean que el aprendizaje de conceptos científicos se aleja, entonces, de la mera superficialidad, es decir, del aprendizaje memorístico, cuando se aplica la metodología científica para aprender un concepto, lo que conlleva al logro de la construcción del conocimiento.

De acuerdo con investigaciones realizadas en el campo de la didáctica de las ciencias, se muestra que existen diferentes dificultades en los procesos de aprendizaje de esta disciplina; dentro de ellas podemos citar las siguientes: la estructura lógica de los contenidos conceptuales, el nivel de exigencia formal de éstos, la falta de preparación de los profesores y la influencia de los conocimientos previos y preconcepciones del alumno. Por ello, es necesario pensar en opciones en las que el desarrollo de los contenidos se enfoque como un planteamiento y que su resolución se desarrolle, en forma conjunta, por parte del profesor y los alumnos.

Podemos afirmar que la investigación dirigida más que un método de enseñanza se constituye en un método de construcción del aprendizaje, el cual brinda a los y las estudiantes las herramientas para el auto-aprendizaje, y llega a la premisa del aprender a aprender, la que genera autonomía y capacidad crítica en el discente investigador (Cañal y Porlan, 1987).

Este modelo por investigación usa el enfoque de problemas entendidos como "una situación incierta que provoca en quien la padece una conducta (resolución del problema) tendiente a hallar la solución (resultado) y reducir de esta forma la tensión inherente a dicha incertidumbre" (Perales, 2000, citado por Ruiz, 2007, p. 52). Los problemas así percibidos permiten diagnosticar ideas y construir nuevos conocimientos, adquirir habilidades de rango cognitivo, promover actitudes positivas hacia la ciencia y actitudes científicas, acercar los ámbitos del conocimiento científico y cotidiano, y evaluar el conocimiento científico del alumno.

Las situaciones problemáticas son preguntas e inquietudes que surgen en la vida cotidiana del educando y que requieren una solución en el momento; pueden ser cerradas (con una sola respuesta) o abiertas para las cuales existen diferentes respuestas o diferentes formas de solución, las que, a la vez, no tienen una solución inmediata y que, por tanto, trascienden la esfera del conocimiento en ese momento; deben ser presentadas en el aula de clase (como mecanismos que promuevan en el educando una reflexión y una confrontación permanente de sus saberes y procedimientos), pues ello facilita el desarrollo de habilidades cognitivas y acerca al educando a procesos conscientes, con los que él mismo demuestra la eficiencia y el alcance de sus acciones.

Es importante destacar que para que este método tenga éxito, se requiere de un docente con un alto dominio disciplinar y un buen manejo pedagógico, que le permita contextualizar los conceptos para que los estudiantes le encuentren sentido al aprendizaje de las disciplinas que se enmarcan en las ciencias. Lo anterior porque la enseñanza de éstas, ha estado muy aislada de la vida cotidiana y no ha habido preocupación por desarrollar actitudes responsables ante los retos morales, sociales, económicos y ambientales que se nos presentan día a día.

\section{Aprendizaje por descubrimiento}

En esta forma de aprendizaje el alumno obtiene el conocimiento descubriendo los principios de la ciencia, por sí mismo. Pero para lograr un proceso de aprendizaje óptimo es necesario desarrollar en el discente algunas habilidades, a saber: la observación, la elaboración de supuestos, la problematización, la clasificación, la organización coherente de la información, la recolección, el análisis de datos y la confrontación para llegar a la obtención de conclusiones (Alfono, 2004). 
Según Pozo y Gómez (1998), una de las mejores formas de aprender es mediante la creación o el descubrimiento por sí mismo, en vez de que otra persona sea la que le transmita ese conocimiento nuevo. Podría decirse que el aprendizaje por descubrimiento consiste en experimentar por sí mismo las cosas nuevas y formarse un concepto propio, fundamentado en sus experiencias, su realidad y su entorno.

El aprendizaje por descubrimiento permite el desarrollo de destrezas metacognitivas, uno de los componentes del aprendizaje al que se le está dando especial atención en las teorías de aprendizaje cognocitivistas. Como dicen Novak y Gowin (1988), la metacognición puede concebirse como una ayuda al aprendizaje, pero también puede y debe constituir un objetivo legítimo de la enseñanza, porque fomenta el aprendizaje significativo. Además, los estudiantes poseen información preliminar y la estructuración cuidadosa del material, lo que les permite descubrir principios importantes que les proporciona un aprendizaje eficaz; pero, para ello, es necesario que los nuevos conceptos se asienten sobre una base sólida.

De acuerdo con Pozo y Gómez (1998), el aprendizaje por descubrimiento debe tener en cuenta los siguientes principios:

- Los docentes proporcionan a los estudiantes los problemas para que sean ellos los que busquen las respuestas.

- Los estudiantes tienen la capacidad de resolver problemas, pero deben ser guiados por los profesores, por medio de una organización de experiencias y actividades didácticas.

- La obtención de un significado como producto exclusivo del descubrimiento creativo.

- La formulación y prueba de una hipótesis antes que, simplemente, leer o escuchar las lecciones del maestro.

- El descubrimiento como una forma de razonamiento inductivo, porque los alumnos pasan de estudiar ejemplos a formular reglas, conceptos y principios generales.

- Los descubrimientos realizados por los estudiantes adquieren significado en su aprendizaje.

Podemos decir, entonces, que el aprendizaje por descubrimiento pretende lograr, en el alumno, un cambio conceptual, un reemplazo de sus concepciones anteriores por otras ideas más próximas al conocimiento científico. Según Pozo y Gómez (1998, p. 286): "Es el alumno quien elabora y construye su propio conocimiento y quien debe tener conciencia de sus limitaciones y resolverlas". El alumno debe aprender a organizar los datos obtenidos del descubrimiento y entrelazarlos con la teoría, así como reflexionar sobre el proceso seguido y los resultados obtenidos.

\section{Aprendizaje por indagación}

En un mundo repleto de productos de la indagación científica, la alfabetización científica se ha convertido en una necesidad: todos necesitamos utilizar la información científica para realizar opciones que se plantean a diario (Garritz, 2006). El propósito fundamental de este modelo es ofrecer al estudiante la oportunidad de que indague cómo la ciencia está presente en todas partes y en todas las actividades humanas, y de cómo la vida cotidiana puede ser utilizada en los procesos de enseñanza y de aprendizaje de esta disciplina. Se pretende que los tópicos sean desarrollados partiendo de ejemplos de la vida diaria, para tener otra mirada del mundo que nos rodea.

La indagación de los modelos científicos y las teorías deben estar relacionadas con los experimentos y con el entorno, para comprender el comportamiento del mundo que nos rodea 
tanto macroscópica como microscópicamente, mediante el descubrir, el compartir y el aprender una visión general del comportamiento físico de la materia, hasta llegar a explicar la estructura fundamental. Vivimos en un mundo globalizado en el que todos necesitamos el acceso al lenguaje científico, de ahí que los procesos de enseñanza y de aprendizaje de las ciencias deben ir orientados hacia la resolución de asuntos y problemas, tanto técnicos como sociales, y no sólo hacia el enseñar principios conceptuales abstractos a los cuales, la mayor parte de los alumnos, no le encuentran la utilidad práctica y, por ello, el aprendizaje se vuelve poco significativo.

De acuerdo con lo anterior, Fensham (2004, citado por Garritz, 2006), dice que el objetivo prioritario de la enseñanza y del aprendizaje de las ciencias debe ser promover una actitud positiva en los estudiantes hacia la ciencia escolar, que mantenga la curiosidad y mejore la motivación con el fin de generar apego y vinculación hacia la educación científica, no sólo durante la época escolar, sino, también, a lo largo de toda la vida. Para ello es necesario llevar al estudiante a la indagación de los fenómenos, de los hechos y de las teorías, entre otros; que le permita realizar observaciones, hacer preguntas, revisar diferentes fuentes de información, contrastar con lo que ya sabe, analizar e interpretar datos, formular respuestas, dar explicaciones y llegar a conclusiones.

Como dicen Posse, Castillo y Páramo (2004), esa curiosidad, ese afán de conocimiento, ese deseo de comprender es la premisa inicial, en cualquier persona, para todo proceso de aprendizaje, lo que lleva a los estudiantes a reclamar una forma diferente de aprender las ciencias, por lo que es necesario desarrollarla de manera que satisfaga e incremente ese afán de conocimiento e investigación, principio básico del aprendizaje para mantenerlo a lo largo de la vida.

Según Garritz (2006), los objetivos de esa nueva educación se contraponen a los que caracterizaron a la educación tradicional de las ciencias, y cita los siguientes:

- Los contenidos se revisten de relevancia personal y social para los aprendices, pues parten de lo que ya saben, de su experiencia previa a la escuela.

- Las habilidades prácticas y el conocimiento tendrán criterios de logros que todos los aprendices puedan alcanzar hasta algún nivel.

- Los temas, los tópicos o las secciones serán visibles, constantemente, para poder aclarar las partes componentes del aprendizaje.

- La pedagogía explotará las demostraciones y las prácticas inherentes a las ciencias y al aprendizaje cultural, el cual se obtiene en forma previa o fuera de la escuela.

- El aprendizaje de habilidades prácticas y cognitivas surgirá como consecuencia fluida de la relevancia y la significatividad de los tópicos de la naturaleza de la ciencia, más que como motivo primario del aprendizaje.

- La evaluación reconocerá tanto los conocimientos previos que los aprendices tienen sobre la ciencia, como sus logros subsecuentes en el resto de los criterios que componen el currículo.

De lo anterior, se puede concluir que la indagación requiere de una metodología que parte del mundo material que nos rodea, mediante preguntas que hay que saber formular y resolver; de acuerdo con Aránega y Ruiz (2005), para ello hay que entrar en la indagación científica, que nos lleva a la identificación de suposiciones, al empleo del razonamiento crítico y lógico y a la consideración de explicaciones alternativas.

En síntesis, el proceso educativo en las ciencias por indagación le permite al estudiante valorar la curiosidad científica y la capacidad de análisis como fuente de aprendizaje, y utilizar el entorno cotidiano como un elemento cercano en la didáctica de las ciencias, idóneo para propiciar aprendizajes significativos. 


\section{La enseñanza de las ciencias y las nuevas tecnologías}

En tiempos de globalización y de cambios rápidos como los que estamos viviendo existen retos para mejorar la educación, que ésta tenga un efecto transformador y que contribuya al desarrollo (Herrera, 2004). Para ello es necesario que los alumnos cuenten con ambientes de aprendizaje más efectivos y didácticos y entornos educativos que les permitan desarrollar sus habilidades para pensar y desarrollar su capacidad de aprender. Pero cuando el diseño de estos ambientes educativos se realiza sin un sustento científico adecuado y sin una propuesta didáctica claramente definida, los beneficios pueden verse disminuidos.

Por ello, los docentes de ciencias debemos tener claridad sobre las directrices que orientan nuestra práctica profesional, de tal forma que se acople a las necesidades de la diversidad y la complejidad. Es decir, el docente debe ver el hecho educativo como un propósito de construcción de sujetos, diversos y creativos en sociedades complejas que basan su desarrollo según el nivel tecnológico (Herrera, 2004). De acuerdo con esta concepción, el docente consolida las actuaciones desde su reflexión y su comprensión de la realidad educativa, y promueve la defensa de su actuación profesional, en la formación humana. De ahí que el saber y la práctica educativa necesitan de una reforma rápida y profunda que permita que las interacciones ciencia, tecnología y sociedad ayuden a la construcción de un mundo más justo.

En décadas anteriores, las preocupaciones curriculares se centraban, casi exclusivamente, en la adquisición de conocimientos científicos, con el fin de familiarizar a los estudiantes con las teorías, los conceptos y los procesos científicos, pero a fines de la década del 90 del siglo xx, con el desarrollo de la Internet, se plantea la idea de diseñar un marco de referencia para la creación de los sistemas educativos desarrollados en la llamada sociedad de la información (Ramírez, 2008). En este contexto, las tecnologías educativas deben adaptarse a las nuevas tecnologías, con el fin de facilitar el acceso de los ciudadanos a la educación, en el marco del desarrollo tecnológico de la informática y de las telecomunicaciones.

En este sentido, el aprendizaje implica un proceso de construcción y reconstrucción en el que las aportaciones de cada estudiante juegan un papel decisivo, y le atribuyen sentido a lo que aprende en relación con su realidad. Es el resultado de un proceso dinámico, individual y social, en el que se construyen conocimientos, se desarrollan valores, actitudes, aptitudes y habilidades, se acomodan y reorganizan nuevos esquemas de conocimiento (modificación de las estructuras cognitivas) que le permiten al estudiante comprender, reconstruir y enfrentar la realidad, y desarrollar sus potencialidades utilizando la tecnología como un medio (Martínez y Ortega, 2009).

Según Ramírez (2008), surge, así, lo que podría considerarse un nuevo paradigma educativo, que enfoca los sistemas de enseñanza desde la ingeniería informática aplicada y el diseño de herramientas de aprendizaje. Esto hace posible que los estudiantes aprendan sobre la ciencia y sobre el mundo natural con múltiples medios y en múltiples entornos de aprendizaje. Pero los procesos de enseñanza y de aprendizaje deben tener una dinámica pedagógica que promueva la apropiación e interiorización del conocimiento, para que el estudiante sea un mediador proactivo y no en un receptor pasivo.

\section{A manera de conclusión}

La ciencia por sí sola no contribuirá a que el mundo sea un lugar mejor, aprender, hechos, teorías, fórmulas y métodos de la investigación científica, entre otros; sin considerar los impactos que tienen en la vida cotidiana no favorecerá a que los estudiantes mejoren sus vidas. Es necesario 
entender cómo la ciencia y, sobre todo, la educación científica pueden alfabetizarnos para ayudar a que todos los habitantes del planeta, logremos un ambiente sano y en equilibrio, con el fin de construir un mundo sostenible y con justicia. La educación científica tiene un gran reto, el cual es posible combatir por medio de un cambio en las formas como se ha enseñado y se ha hecho ciencia hasta ahora, para potenciar nuevas formas de pensar, enseñar y aprender.

Podría afirmarse que algunas de las razones del desinterés de los estudiantes hacia el estudio de las ciencias, es la poca relación que existe entre la manera como se enseña y la vinculación con el mundo que los rodea, su falta de aplicaciones prácticas y la poca relación entre la ciencia, la tecnología, la sociedad y el proceso educativo. Por ello, es necesario que los modelos sobre la enseñanza y el aprendizaje de las ciencias, en términos generales, tomen en cuenta algunos factores, entre ellos: las características socioculturales y cognitivas de los alumnos, sus concepciones epistemológicas y destrezas metacognitivas, las relaciones en el aula, los aspectos relacionados con la motivación, los recursos y, sobre todo, el contexto.

La didáctica de las ciencias tiene la responsabilidad de provocar profundos cambios en los diferentes elementos del currículum y la metodología de la enseñanza, con el fin de lograr: que los cursos se desarrollen vinculados con la realidad y que los estudiantes aprendan lo indicado, para poseer una alfabetización científica que les sirva para la vida. Para ello, los docentes necesitan una didáctica coherente y adecuada al actual contexto sociocultural, que les permita una formación científica apropiada para las nuevas generaciones. Como decía Freire (2002): el hombre es hombre, y el mundo es mundo, en la medida en que ambos se encuentren en una relación permanente, el mundo será transformado.

\section{Referencias bibliográficas}

Alfono, C. (2004). Familiarización de los estudiantes con la actividad científica investigadora: Método dinámico para caracterizar el movimiento de traslación de un cuerpo. Revista Enseñanza de las Ciencias, 3(1), 1-13.

Aránega, R. \& Ruiz, M. (2005). Indagar en el entorno cotidiano: clave para la formación científica de los educadores. Revista Enseñanza de las Ciencias, VII, 1-4 (número extraordinario, CONGRESO).

Campanario, J. M. \& Moya, A. (2002). ¿Cómo enseñar ciencias? Principales tendencias y propuestas. Revista Enseñanza de las Ciencias, 17, 179-192.

Cañal P. \& Porlán, R. (Coords.). (1987). ¿Qué enseñar? V Jornadas de Estudio sobre la Investigación en la Escuela. Realizadas en Sevilla, del 5 al 7 de diciembre, 1987.

Garritz, A. (Set.-Dic., 2006). Naturaleza de la ciencia e indagación: cuestiones fundamentales para la educación científica del ciudadano. OEI Revista Iberoamericana de Educación, 42, 127-152.

Diego-Rasilla, F. J. (2004). El método científico como recurso pedagógico en el bachillerato: Haciendo ciencia en clase de Biología. Pulso, 111-118.

Diéguez, L. A. (1993). Cientifismo y modernidad: Una discusión sobre el lugar de la ciencia. (Rubio Carracedo, editor) El giro posmoderno, suplemento $\mathrm{N}^{\mathrm{o}} 1$ de Philosophica Malasitana, 81-102. 
Furió, C. \& Guisasola, J. (1999). Concepciones alternativas y dificultades de aprendizaje en electrostática. Selección de cuestiones elaboradas para su detección y tratamiento, Enseñanza de las Ciencias, 17(3), 441-452.

Freire, P. (1995). Pedagogía del oprimido (20ª ed.). Madrid, España: Siglo XXI editores.

Freire, P. (2002). La educación como práctica de la libertad. Madrid, España: Siglo XXI editores.

Herrera, M. (2004). Las nuevas tecnologías en el aprendizaje constructivo. OEI. Revista Iberoamericana de educación, 34(4), 1-19.

Huffmann, D. (2005). ¿Qué es la ciencia? Antología del curso Filosofía de la ciencia. Doctorado en Ciencias Naturales para el Desarrollo. Programa Inter-universitario: ITCR, UNA y UNED de Costa Rica; UNAM y UACh de México; UNAN de Nicaragua. Doctor de la Universidad Autónoma de Chapingo. Documento inédito. Chapingo, México.

Martínez, A. \& Ortega, J. (2009). Educación científica de calidad basada en una tecnología oportunamente estratégica. Revista Electrónica Química Viva, Vol. 8(1), 48-55.

Morin, E. (2000). El paradigma antológico. Recuperado el 3 de julio de 2008, de http://www. ujaen.es/dep/derpen/filmoral/pdi/articulos/EL\%20PARADIGMA\%20\%20\%20\%20\%20\%20 ECOLOGICO.doc

Morin, E. (2001). Los siete saberes necesarios para la educación del futuro. Barcelona, España: Editorial Paidós.

Novak, J. D. \& Gowin, D. B. (1988). Aprendiendo a aprender. Barcelona, España: Martínez Roca.

Núñez, J. (2000). Lo que la educación científica no debería olvidar: Rigor, objetividad y responsabilidad social. Recuperado el 16 de marzo de 2005, de http://www.campus-oei.org/salactsi/ $\underline{\text { nunez05.htm }}$

Posse, P., Castillo, D. \& Páramo, E. (2004). El método como curiosidad. Cuadernos de Pedagogía, Vol. 340, 60-63.

Pozo, J. I. \& Monereo, C. (Eds.). (1999). El aprendizaje estratégico. Enseñar a aprender desde el currículo. Madrid, España: Santillana/Aula XXI.

Pozo, J. \& M. Gómez. (1998). Aprender y enseñar ciencia: Del conocimiento cotidiano al conocimiento científico. Madrid, España: Ediciones Morata.

Ramírez, L. (Abr. 2008). El nuevo paradigma de la Educación Superior a Distancia. Algunos criterios para el porvenir. Revista Cognición $N^{o}$ 13, 18-40. Edición especial II CONGRESO CREAD ANDES y II ENCUENTRO VIRTUAL EDUCA UTP, Loja, Ecuador.

Ruiz, O. F. (Jul.-Dic. 2007). Modelos didácticos para la enseñanza de las ciencias naturales. Revista Latinoamericana de Estudios Educativos, 3(2), 41-60. 
Tejada, J. (2005). Didáctica-curriculum, diseño, desarrollo y evaluación curricular. Barcelona España: Editorial Davinci.

Toledo, U. (1998). La epistemología según Feyaberand. Cinta de Moebio No. 4 Recuperado el 4 de abril de 2005 de http://rehue.csociales.uchile.cl/publiaciones/moebio/04/frames01.htm 